\title{
OA02 I-0 I. Construction and characterization of replication competent attenuated NYVAC-based vectors as potential HIV vaccines
}

\author{
B Jacobs*1, K Kibler ${ }^{1}$, S Wong11, T Huynh ${ }^{1}$, S Holechek1 ${ }^{1}$ K Denzler ${ }^{1}$, \\ W Arndt ${ }^{1}$, M Parrington ${ }^{2}$, J Tartaglia ${ }^{2}$ and G Pantaleo ${ }^{3}$
}

Address: ${ }^{1}$ The Biodesign Institute, CIDV, Arizona State University, Tempe, AZ, USA, ${ }^{2}$ Sanofi-Pasteur, Toronto, Canada and ${ }^{3} \mathrm{CHUV}$, Lausanne, Switzerland

* Corresponding author

from AIDS Vaccine 2009

Paris, France. 19-22 October 2009

Published: 22 October 2009

Retrovirology 2009, 6(Suppl 3):OI3 doi:I0.1 I86/I742-4690-6-S3-OI3

This abstract is available from: http://www.retrovirology.com/content/6/S3/OI3

(C) 2009 Jacobs et al; licensee BioMed Central Ltd.

\section{Background}

Poxvirus viruses have been utilized for many years as vaccine vectors. Recent years have seen an increase in efforts to identify safer pox vectors to express heterologous antigens, in this case HIV antigens.

Safer pox vectors have included MVA, ALVAC and NYVAC, which are all replication deficient in human cells. However, the loss of replication competence has also reduced antigen expression, and therefore, immunologic response to the vaccination. We have constructed vectors in a NYVAC background that are replication competent but attenuated for virulence.

\section{Methods}

To restore replication competence in NYVAC that expresses clade C HIV gag, pol, nef and env (NYVAC-C), we inserted genes deleted in the parental vector: 1) NYVAC-C-KC includes the human host range genes $K 1 \mathrm{~L}$ and $C 7 L$; 2) NYVAC-C+12 includes the entire cassette of genes from $K 1 L$ to $C 7 L$ that is deleted from NYVAC.

To decrease virulence, we deleted the $E 3 L$ gene, which is required for interferon-resistance and virulence, and replaced it with a gene from Ambystoma tigrinum virus (ATV, the new virus is NYVAC-C+12-ATV), which restores a single round of replication.

\section{Results}

In vitro characterization of the constructs demonstrates restoration of replication in primary and human cell lines. NYVAC-C+12-ATV leads to induction of pro-inflammatory signal transduction pathways. Pathogenicity studies in newborn mice demonstrate attenuation of 3-5 logs when compared to wt vaccinia virus or to NYCBH.

\section{Conclusion}

NYVAC constructs provide increased safety in the most sensitive of animal models. Because antigen expression is increased in these replication-competent constructs, they may be more immunogenic than replication-defective poxvirus vectors. Increased pro-inflammatory signal transduction in cells infected with NYVAC-C+12-ATV may also lead to an increase in immunogenicity of this highly attenuated vector. Thus, these replication competent, attenuated viral vectors may have improved immunogenicity over replication defective poxvirus vectors, while maintaining an improved safety profile. 